\title{
Short-Term Hydro Generation Scheduling Of The Three Gorges Hydropower Station Using Improver Binary-coded Whale Optimization Algorithm
}

Kun Yang ( $\sim$ yangkun961249@126.com )

Hohai University

Kan Yang

Hohai University

\section{Research Article}

Keywords: Short-term hydropower generation scheduling, Whale optimization algorithm, Adaptive inertia weight, Perturbation mutation strategy, Optimal stable load distribution table

Posted Date: March 22nd, 2021

DOI: https://doi.org/10.21203/rs.3.rs-159194/v1

License: (c) (i) This work is licensed under a Creative Commons Attribution 4.0 International License.

Read Full License

Version of Record: A version of this preprint was published at Water Resources Management on July 31st, 2021. See the published version at https://doi.org/10.1007/s11269-021-02917-0. 
Title: Short-Term Hydro Generation Scheduling Of The Three Gorges Hydropower Station Using Improver Binary-coded Whale Optimization Algorithm

\title{
Author information
}

\section{Affiliations:}

College of Hydrology and Water Resources, Hohai University, 1\# Xikang Road, Gulou District, Nanjing 210098, China.

Kun Yang \& Kan Yang

Contributions: Conceptualization and material preparation: Kan Yang; Methodology, data collection, modelling process and analysis: Kun Yang.

Corresponding author: Correspondence to Kan Yang. Email: kyang@hhu.edu.cn.

\begin{abstract}
An improved binary-coded whale optimization algorithm (IBWOA) is proposed to solve the complex nonlinear problem of short-term hydropower generation scheduling (STHGS). The spatial optimal load distribution is combined with the temporal unit commitment combination model, and the binary array is used to represent the start/stop state of the unit. Sigmoid Function (SF) is used to solve the correspondence between binary array and real number. The whale algorithm's search mechanism is optimized, and the inertia weight and perturbation variation strategy are introduced to improve the algorithm's optimization ability. The unit commitment (UC) subproblem was solved by repairing the minimum uptime/downtime constraint and the spinning reserve capacity constraint, and the economic load scheduling (ELD) subproblem was solved by an optimal stable load distribution table (OSLDT). The Mutation mechanism and the Locally balanced dynamic search mechanism compensate for the non-convex problems caused by start-stop constraints and stable optimal table methods. The proposal is applied to solve the STHGS of the Three Gorges hydropower station. The results show that the method has good convergence, stability, fast calculation speed, and high optimization accuracy.
\end{abstract}


Keywords: Short-term hydropower generation scheduling; Whale optimization algorithm; Adaptive inertia weight; Perturbation mutation strategy; Optimal stable load distribution table 


\section{Introduction}

Hydropower is an ideal renewable and clean resource with many advantages, such as pollution-free, low operating cost, and strong peak-regulating capacity. Nowadays, people's demand for electricity is huge, so water energy's effective use has become essential water conservancy research. It is one of the keys to the utilization of water energy that hydropower stations are run economically. A fair distribution of a load of hydropower units can increase the power generation and protect unit components' long-term stability.

Short-term hydropower dispatch (STHGS) of hydropower station is a daily optimization operation problem of the power system, which aims to minimize the total water consumption of the unit and the startup/shutdown costs of the unit while meeting the grid demands and other hydropower constraints (Cristian et al. 2013; Ohishi T et al. 2005). The STHGS is a complicated problem that mainly includes two parts (Peng et al. 2015): one is the external unit combination (UC) sub-problem, the other is the internal economic load distribution(ELD) sub-problem. The process to solve the UC problem is to formulate the start-stop rules for the unit and to open/close the unit under specific load requirements according to the characteristics of the unit. The UC sub-problem needs to avoid frequent on/off operations, which can not only reduce the water consumption in the process of starting and closing the hydropower unit (Nilsson and Sjelvgren 1997), but also increase the life of the unit. The process to solve the ELD problem is to allocate the load to the starting unit to obtain the minimum water consumption under the condition that the total load meets the requirements. The total water consumption of hydropower stations is minimized by considering the proper online and offline dispatching of each unit, the reasonable distribution of each operating unit's load, and the constraints of various equations and inequalities. (Wood and Wollenberg 1996). The operation of hydropower stations needs to meet a series of complex constraints, such as power balance constraints, unit operating conditions constraints, spinning reserve capacity constraints, and minimum uptime/downtime constraints (Finardi and Scuzziato 2013). 
A variety of mathematical methods have been developed to solve the STHGS problem. Traditional algorithms include lagrangian relaxation (LR) (Cheng et al.2000), linear programming (LP) (Jabr et al. 2000), nonlinear programming (Catalão et al. 2006), and dynamic programming (DP) (Wang et al.1993; Siu et al. 2001; Pérez-Díaz et al. 2010). These techniques have received different degrees of success and have been widely accepted in the power industry. However, these methods have some disadvantages in solving the STHGS problem. LR can use the Lagrange multiplier to establish dual problems to simplify calculations, but it is challenging to find suitable Lagrange multipliers. The linear programming method is inefficient and cannot handle complex STHGS problems with nonlinear constraints. Although DP can find the optimal scheduling table of STHGS problem, it suffers from the "curse of the dimensionality" when facing large-scale units and multiple time scales (Labadie 2004; Zhao et al. 2012). Incremental dynamic programming algorithm(IDP) (Jukna 2014) and progressive optimality algorithm (POA) (Feng et al. 2018) can overcome this shortcoming; however, these algorithms require convex objective functions (Lu et al. 2013). Therefore, with the expansion of the scale of hydropower stations and the increase in generating units' capacity, traditional methods are incapable of solving the problem.

Therefore, a variety of heuristic optimization algorithms have been proposed for the STHGS problem in order to deal with the complex problem of multi-constraint nonlinearity. These are genetic algorithm (GA) (Senthil and Mohan 2010; Zheng et al. 2013), particle swarm optimization (PSO)(Kumar et al. 2011; Fakhar et al. 2015), chaotic optimization algorithm (COA) (Cai et al. 2007), ant colony optimization (ACO) (Shi et al. 2004), simulated annealing (SA)(Dudek et al. 2010; Saraiva et al. 2011), bee colony optimization (BCO) (Peng et al. 2015), and bat algorithm (BA) (Su et al. 2019). These algorithms have better performance than traditional mathematical methods in computational precision, efficiency, and reliability. However, they also have inevitable defects and defective parts. GA suffers from high randomness and blindness and can easily produce premature 
phenomena (Shang et al. 2019). The ant colony algorithm can be combined with an equal incremental method(Hu et al. 2012), but the disadvantage of this method is that with the increase of hydropower units and parameter settings, the calculation speed becomes slower, and the algorithm is easy to converge prematurely. Chen and Guo (2011) and Wu (2015) adopt improver discrete particle swarm optimization (DPSO) to solve the UC problem. However, the increase in the number of hydropower units would also lead to low global convergence efficiency. Hu et al. (2019) proposed social spider optimization (SSO), and Yang et al. (2020) proposed a discrete shuffled frog leaping algorithm(DSFLA) for solving STHGS. However, these algorithms require many parameters, which are more difficult to optimize in complex STHGS. As a result, the convergence efficiency of the algorithm is easily affected by the quality of the parameters. Therefore, a whale algorithm with low parameter requirement and good optimization effect is proposed to solve this problem.

Whale optimization algorithm(WOA), a novel algorithm, was proposed by Mirjalili and Lewis in 2016 (Mirjalili and Lewis, 2016). The algorithm was inspired by way of whales hunt. The primary process includes three stages: surrounding prey, bubble-net attack, and random search. The whale algorithm has been applied in many different fields by scholars in recent years due to its advantages, such as fewer parameters, strong optimization ability, and fast optimization speed. The whale algorithm's search space is applicable to continuous-value positions, so it cannot be used to solve the STHGS problem because the start/stop state of the unit is often expressed by 0 and 1 . Thus, this paper proposes an improved binary-coded whale optimization algorithm (IBWOA) to solve the problem of UC. A whale population is established in IBWOA, and each value of the whale represents a shortterm generation scheduling unit. The whale algorithm's search mechanism is improved so that the problem that the algorithm is easy to fall into the local optimum is improved. The nonlinear inertia weight is introduced to improve the optimization speed of the algorithm. For the UC problem, the improved sigmoid function is used to limit individual whale value to a range of $0-1$, and the binary array of $0-1$ is obtained according to its probability to represent the start/stop state of the unit. The 
UC subproblem was solved by repairing the minimum uptime/downtime constraint and the spinning reserve capacity constraint to the derived solutions of each generation satisfy the constraint requirements and avoid a large number of inferior solutions derived due to the randomness of the algorithm. The ELD subproblem was solved by the stable optimal table obtained from a dynamic programming method so that the derived solutions of each generation can satisfy the load requirements. Finally, the locally balanced dynamic search mechanism is introduced to optimize the local solution and get the final load allocation scheme. These strategies significantly reduce calculation time and efficiency. In this paper, IBWOA is applied to thr Three Gorges Hydropower Station, and the results show that the algorithm has high effectiveness and feasibility.

\section{The Economical Operation Problem Formulation}

\subsection{Objective Function}

The emphasis of economic operation in hydropower stations is the optimal distribution of load. It means that load requirements should be formulated according to the user's electricity consumption, and reasonable load distribution of the unit should be formulated on the premise of safety to find the minimum water consumption of power generation. The formula is as follows:

$$
W=\min \sum_{t=1}^{T} \sum_{i=1}^{I}\left[u_{i, t} Q_{i, t}\left(H_{i, t}, P_{i, t}\right) \Delta t+u_{i, t}\left(1-u_{i, t-1}\right) Q_{i}^{u p}+u_{i, t-1}\left(1-u_{i, t}\right) Q_{i}^{d o w n}\right]
$$

where $\mathrm{W}$ denotes total water consumption of power generation; $Q_{i, t}\left(H_{i, t}, P_{i, t}\right)$ denotes the outflow of ith unit at time $\mathrm{t}$ when the output is $P_{i, t}$ and the water head is $H_{i, t} ; u_{i, t}$ and $u_{i, t-1}$ are the online/offline state of the ith unit at time t and t-1, respectively; $Q_{i}^{\text {up }}$ and $Q_{i}^{\text {down }}$ are the water consumption of the ith unit when the unit startup and stop, respectively; $\Delta t$ is the time interval; I is the total number of units; $\mathrm{T}$ is the total number of time;

\subsection{Constraints Condition}

The STHGS problem is a multi-constraint problem. In terms of safety, there are unsafe load ranges of the generator unit called cavitation-vibration regions. If the generator unit operates in this region for a long time, it will cause severe damage the generator unit. Second, the STHGS also needs to 
satisfied Power balance constraints, Power generation limits constraint, Minimum uptime/downtime constraint, Spinning reserve capacity constraint, and water balance constraints. The constraints to be satisfied in the mathematical model are as follows:

(1) Power balance constraint

$$
P d_{t}=\sum_{i=1}^{I} u_{i, t} P_{i, t}
$$

where $P d_{t}$ is the load conditions undertaken by a hydropower station; $P_{i, t}$ and $u_{i, t}$ are output and the online/offline state of the ith unit at time $t$, respectively.

(2) Power generation limits constraint

$$
N_{i, \min } \leq P_{i, t} \leq N_{i, \max }
$$

where $N_{i, \min }$ and $N_{i, \max }$ are the lower and upper output bounds of the ith hydropower unit, respectively.

(3) Spinning reserve constraint

$$
P d_{t}+L R_{t}^{\min } \leq \sum_{i=1}^{I} u_{i, t} P_{i, t}^{\max } \leq P d_{t}+L R_{t}^{\max }
$$

where $L R_{t}^{\min }$ and $L R_{t}^{\max }$ signify lower limit and upper limit of the spinning reserve capacity at time $\mathrm{t}$, respectively.

(4) Minimum uptime/downtime constraint

$$
\left\{\begin{array}{l}
T_{i, t}^{o n} \geq T_{i}^{u p} \\
T_{i, t}^{o f f} \geq T_{i}^{\text {down }}
\end{array}\right.
$$

where $T_{i, t}^{o n}$ and $T_{i, t}^{o f f}$ are the duration of uptime/downtime that the ith hydropower unit, respectively; $T_{i}^{u p}$ and $T_{i}^{\text {down }}$ the shortest time limits of startup and shutdown that the ith hydropower unit, respectively.

\section{The traditional WOA Algorithm}

The traditional WOA algorithm simulates the predation behavior of humpback whales. Humpback 
whales hunt their prey in three ways: random search, surrounding the prey, and bubble-net attack. The random search method means that one whale is randomly selected as the target, and all the whales are close to it so that the whale can hunt for its prey in a wide range. The method of encircling prey means that all whales move towards individuals with the highest fitness. This method allows all whales to approach their prey quickly. The bubble net attack method means that all whales move in a spiral direction toward their prey, which allows them to search all areas around their prey. Mathematical models of the three behaviors are established as follows:

\subsection{Searching for prey}

The formula is as follows:

$$
\begin{gathered}
X(t+1)=X^{*}(t)-A \cdot D \\
D=\left|C \cdot X^{*}(t)-X(t)\right| \\
A=2 a \cdot r-a \\
C=2 \cdot r \\
a=2-2 \cdot t / M
\end{gathered}
$$

where $\mathrm{t}$ is the number of current iterations, $\mathrm{M}$ is the maximum number of iterations; $X^{*}(t)$ is the best individual in the current population; $X(t)$ is the location of individual whales, $\mathrm{r}$ is random numbers between 0 and 1 ; $a$ is parameters between 2 and 0 .

\subsection{Bubble net attack}

Humpback whales feed by spiraling, and the formula is as follows:

$$
\begin{gathered}
X(t+1)=D \cdot e^{b l} \cdot \cos (2 \pi l)+X^{*}(t) \\
D=\left|X^{*}(t)-X(t)\right|
\end{gathered}
$$

where $\mathrm{D}$ is the difference between the current individual and the optimal solution; $\mathrm{b}$ is control parameters; 1 is random Numbers between -1 and 1 . 


\section{3 random search method}

The formula is as follows:

$$
\begin{gathered}
X(t+1)=X_{\text {rand }}-A \cdot D \\
D=\left|C \cdot X_{\text {rand }}-X(t)\right|
\end{gathered}
$$

where $X_{\text {rand }}$ is randomly selected individuals. If $A \geq 1$, the herd of whales moves randomly toward anyone whale. This method can make the algorithm escape from the current optimal solution temporarily.

\section{Methodology of improved binary-coded whale optimization (IBWOA) algorithm}

\subsection{Improving the random search method}

The optimization ability of the algorithm is guaranteed due to three predation behaviors of WOA. However, these three behaviors affect each other. When the weight of the random search is larger, the convergence efficiency of the algorithm is lower. This algorithm will be precocious if the weights of the other two methods increase. These are not conducive to the global optimization of the algorithm.

According to the calculation formula, the whale algorithm will stop the random search mode when $\mathrm{t}$ is $2 / \mathrm{M}$. However, the random search method and the surrounding method would be equally weighted if we set the condition that $\mathrm{A}=0$. This method will make the algorithm lose optimization efficiency. So the improved random search condition theory is proposed. The formula is as follows:

$$
q=q_{2}+\left(q_{1}-q_{2}\right)\left(\frac{t}{M}\right)
$$

where $\mathrm{q}$ is random search probability; $q_{1}$ and $q_{2}$ are the maximum and minimum probability of random search, respectively, and $q_{1} \geq q_{2} \geq 0.5$. A random number $\mathrm{p}$ from 0 to 1 is generated during the algorithm operation. The value of A determines whether to conduct a random search when $p<0.5$. Otherwise, calculate $\mathrm{q}$ according to the number of iterations, and whales conduct a random search when $p \geq q$. This method ensures that the algorithm has global search capability over the 
whole period.

\subsection{Adaptive Nonlinear Inertia Weight}

A standard method to improve intelligent optimization algorithms is inertia weight. This method can balance the early global optimization ability and the algorithm's late local optimization ability by changing the search space. The traditional inertia weight method makes the search space decrease linearly, but the optimization effect is usually not ideal. Therefore, an adaptive nonlinear inertia weight is proposed. The formula is as follows:

$$
\begin{gathered}
w=w_{1}-\left(w_{1}-w_{2}\right)\left(\frac{t}{M}\right)^{\frac{1}{t}} \\
X(t+1)=\left\{\begin{array}{lc}
X^{*}(t)-w \cdot A \cdot D & \mathrm{p}<0.5 \\
w \cdot D \cdot e^{b l} \cdot \cos (2 \pi l)+X^{*}(t) & \mathrm{p} \geq 0.5
\end{array}\right.
\end{gathered}
$$

Where $w_{1}$ and $w_{2}$ are the maximum and minimum values of the weight parameters; $X^{*}(t)$ is the best individual position; $X(t+1)$ is the updated position of the individual.

\subsection{Mutation mechanism}

WOA already has good search efficiency and few parameter requirements. The above adaptive inertia weight and the improved random search strategy have effectively balanced the algorithm's development and mining process. However, the two results obtained by Sigmid transform may have high fitness when solving a 0-1 problem. The STHGS problem is a complex problem with multiple constraints, so several steps follow to handle the constraints, which can cause the solution to be nonconvex.

A mutation mechanism is designed to increase the population diversity to avoid the algorithm falling into the local optimum. The constraint conditions of the model include uptime/downtime repair strategy and spinning reserve capacity constraints, which relate to the running time and start-stop sequence of the unit, so scheduling tables with good results may be ignored due to violation of constraints. It is essential to design an evolutionary strategy that does not violate constraints for 
solving STHGS problems. The uptime/ downtime sequence table is selected according to the water consumption in the stable operation area of the unit. However, there are many stable operation areas, and the average water consumption cannot represent all load areas. Also, the operation of the units is independent of each other, and the start-stop state of each unit does not affect the minimum start-stop constraint of other units. Therefore, the mutation mechanism is designed to exchange all genes of the two mutation points (representing the unit) (genes represent the unit's on-off schedule). This method does not violate the constraints and increases the diversity of the population, which avoids the algorithm falling into the local optimum. The formula is as follows:

$$
\left\{\begin{array}{l}
M=X_{i_{1}, j}^{t} \\
X_{i_{1}, j}^{t}=X_{i_{2}, j}^{t} \\
X_{i_{2}, j}^{t}=M
\end{array}\right.
$$

where $\mathrm{M}$ is the variables used for temporary storage; $X_{i_{1}, j}^{t}$ and $X_{i_{2}, j}^{t}$ represent the value of population $\mathrm{j}$, time $\mathrm{t}$, unit $i_{1}$, and unit $i_{2}$, respectively. A variation rate $\mathrm{k}_{\mathrm{p}}$ is set before the calculation. A random number between 0 and 1 is calculated for each unit after each iteration. If the random number is less than $\mathrm{k}_{\mathrm{p}}$, the values of the unit at all times are exchanged with the values of another random unit.

\subsection{Adaptive binary-coded theory}

The UC subproblem is a discrete $0-1$ assignment problem, but WOA is suitable for continuous space solutions. Therefore, an improved sigmoid function is used to convert the continuous value of the whale to 0 or 1 according to its probability. The formula is as follows:

$$
\begin{gathered}
y_{i}^{k}=k_{a} \cdot\left[\left(1+e^{-k_{b} \cdot x_{i}^{k}}\right)^{-1}-k_{c}\right] \\
u_{i}^{k}= \begin{cases}1 & \text { if } y_{i}^{k}>\text { rand } \\
0 & \text { if } y_{i}^{k} \leq \text { rand }\end{cases}
\end{gathered}
$$

where $y_{i}^{k}$ is the value corresponding to $x_{i}^{k}$, which ranges from 0 to 1 ; rand is the random number between 0 and 1 . 


\section{Proposed IBWOA for STHGS}

\subsection{Introduction of IBWOA for the UC Sub-Problem}

The whale algorithm cannot be applied to unit combinations because it only works for continuous values. Therefore, the solution needs to be binary encoded. The real value of the solution is mapped between 0 and 1 according to Eq. (19). The unit state is then set to 0 or 1 according to Eq. (20). The matrix representation of the solution is as follows:

$$
X_{i}^{k}=\left[\begin{array}{llll}
x_{i, 1,1}^{k} & x_{i, 1,2}^{k} & \ldots & x_{i, 1 m}^{k} \\
x_{i, 2,1}^{k} & x_{i, 2,2}^{k} & \ldots & x_{i, 2, m}^{k} \\
\ldots & \ldots & \ldots & \ldots \\
x_{i, n, 1}^{k} & x_{i, n, 2}^{k} & \ldots & x_{i, n, m}^{k}
\end{array}\right] \stackrel{{ }_{E q .(20)}}{\longrightarrow} U_{i}^{k}=\left[\begin{array}{llll}
u_{i, 1,1}^{k} & u_{i, 1,2}^{k} & \ldots & u_{i, 1, m}^{k} \\
u_{i, 2,1}^{k} & u_{i, 2,2}^{k} & \ldots & u_{i, 2, m}^{k} \\
\ldots & \ldots & \ldots & \ldots \\
u_{i, n, 1}^{k} & u_{i, n, 2}^{k} & \ldots & u_{i, n, m}^{k}
\end{array}\right]
$$

where $X_{i}^{k}$ is the value of whale; $U_{i}^{k}$ is the start/stop state of the unit.

\subsection{The method for unit combination sub-problem}

Because of the randomness of the optimization algorithm, the feasibility of the original solution will be lost. The algorithm's optimization ability will decline rapidly, and it is challenging to obtain feasible solutions if no constraints are imposed. Thus, two repair mechanisms are used, one is the minimum uptime/downtime constraint, and the other is the spinning reserve capacity constraint. The result of the algorithm is always a feasible solution by using these two repair mechanisms to be optimized within the feasible region.

\subsubsection{A repair strategy for Minimum Uptime/Downtime Constraint}

Because the algorithm's binary results in each iteration are random, each iteration is likely to break the minimum uptime/downtime constraint. Therefore, a minimum uptime/downtime repair strategy is proposed. In this method, the unit which can not meet the constraint will be forced to change its state to obtain the feasible solution that meets the requirement. As shown in fig.1, set the minimum uptime/downtime time to $2 \mathrm{~h}$. Each unit is traversed in chronological order. The array (a) will be converted to the array (b). If the ith unit of time $t$ can not meet the minimum uptime/downtime constraint, its state is forced to change by eq.(22). Otherwise, its unit status is updated by eq. (23). 


$$
\begin{gathered}
u_{i}^{k}= \begin{cases}1 & \text { if } T_{i, t}^{o n}<T_{i}^{u p} \\
0 & \text { if } T_{i, t}^{\text {off }}<T_{i}^{\text {down }}\end{cases} \\
u_{i}^{k}=\operatorname{round}(\text { rand })
\end{gathered}
$$

where rand is the random number between 0 and 1 . If the rand is less than 0.5 , start the unit. Otherwise, close the unit.

\subsubsection{A repair strategy for Spinning Reserve Capacity Constraint}

When the state of the unit is changed, the spinning reserve capacity constraint may be violated. Therefore, the priority list of unit startup/shutdown and dynamic repairing techniques are used to solve this problem. First, the hydropower units' priority table is determined according to the average water consumption in the stable operation area. The calculation formula of the average water consumption is as follows:

$$
\bar{W}_{i}=\frac{1}{L} \sum_{l=1}^{L} \frac{Q_{i}\left(P_{l}\right)}{P_{l}}
$$

where $\bar{W}_{i}$ is the average water consumption of ith unit; $\mathrm{L}$ is the discrete number of stable operation zone; $P_{l}$ is the load at the lth discrete point; $Q_{i}\left(P_{l}\right)$ is the water consumption when the load is $P_{l}$.

In the IBWOA algorithm, if the unit cannot meet the spinning reserve constraint after the uptime/downtime constraint is repaired, the spinning reserve constraint is repaired according to the following rules.

Step 1: The load capacity of all of the committed units in each period is calculated, and the unit is checked whether it meets the current spinning reserve constraint according to Eq.(4).

Step 2: If the online unit's total output is greater than the sum of the power demand and the maximum spinning reserve capacity, the committed units that meet the minimum uptime time are searched in order according to the priority list of unit startup/shutdown. These committed units should be shut down until the load requirements are met. 
Step 3: If the online unit's total output is less than the sum of the power demand and the minimum spinning reserve capacity, the uncommitted units that meet the minimum shutdown time are searched in reverse order according to the priority list of unit startup/shutdown, than these uncommitted units should be opened until the load requirements are met.

Step 4. Suppose there is no unit meeting the minimum uptime/downtime time after the search. In that case, the corresponding unit is forcibly opened/shutdown to force the hydropower station to meet the spinning reserve constraint.

\subsection{OSLDT to Solve the ELD Sub-Problem}

The OSLDT is used to solve the problem of economic load distribution. The OSLDT is the optimal load distribution schedule calculated under a specific load requirement. However, it takes much time to solve the load distribution, and the constraint is very complicated when we combine the UC problem. In order to simplify the calculation, the OSLDT obtained by dynamic programming(DP) under cavitation vibration is prepared, which significantly improves the quality of the solution and reduces a lot of calculation time.

\subsubsection{Establish OSLDT}

The OSLDT is formulated by the DP. The load increases integer from 0 until all units are full capacity. When the assigned load is in the unstable operating zone of the unit, a large penalty function value is applied to avoid the distribution of output in this zone. The minimum water consumption and distribution scheme of each given load is recorded, and the stable optimal scheduling table is obtained. The state transfer equation is shown as follows:

$$
N p_{i}=N p_{i-1}+Z p_{i}
$$

where $N p_{i}$ is the total output of ith; $Z p_{i}$ is the decision variable denoting output of new startup unit at the ith stage.

The recursive equation of the ELD is shown as follows:

$$
Z Q\left(N p_{i}\right)=\operatorname{Min}_{N p_{i} \in C_{i}}\left[Q_{i}\left(Z p_{i}\right)+Z Q\left(N_{i-1}\right)\right]
$$


where $Z Q\left(N p_{i}\right)$ refers the total water consumption of all of the online units when the total output is $N p_{i} ; Q_{i}\left(Z p_{i}\right)$ refers the water consumption of the new starup unit at stage $\mathrm{I} ; c_{i}$ is unit stable operation region.

\subsubsection{Load distribution}

The stable optimal table is formulated by the dynamic programming method. The load increases integer from 0 until all units are full capacity. When the assigned load is in the unit's unstable operating zone, a large penalty function value is applied to avoid the distribution of output in this zone. The minimum water consumption and distribution scheme of each given load is recorded, and the stable optimal scheduling table is obtained.

$$
\begin{gathered}
N z_{j}(k)=\sum_{i=1}^{I} u_{i}^{j} \cdot N p_{i}(k) \\
P_{i}^{j}=N p_{i}(k-1)+\frac{N_{d e}^{j}-N z_{i}^{j}(k-1)}{N z_{i}^{j}(k)-N z_{i}^{j}(k-1)} \cdot\left[N p_{i}(k)-N p_{i}(k-1)\right]
\end{gathered}
$$

where $N z_{j}(k)$ represents the sum of loads allocated by the kth row stable optimal table for current scheduling in the jth period; $N p_{i}(k)$ represents the ith unit load in the stable optimal table at row k; $P_{i}^{j} \quad$ represents the load distribution of unit i in the jth period. $N_{d e}^{j}$ represents the total load required for the jth period.

\subsection{Locally balanced dynamic search mechanism}

Scheduling table satisfying load constraint can be solved quickly according to OLDT, and the obtained solution has high fitness. However, the result is the optimal unit combination under the stable optimal table scheduling because the stable optimal table is adopted. So the load of the unit still needs to be redistributed after the two repair strategies. When the load constraint is satisfied, the locally balanced dynamic search mechanism is used to optimize the load distribution. The formula is set up as follows:

$$
Q_{s}(i, j)=Z Q\left(N_{i}+1\right)+Z Q\left(N_{j}-1\right)
$$




$$
Q_{\text {plan }}(i)=\min \left(Q_{s}(i, j)\right)
$$

where $Q_{s}(i, j)$ represents represents the sum of the water consumption calculated by reducing the ith unit by one unit and adding the $\mathrm{j}$ unit by one unit; $N_{i}$ and $N_{j}$ represent the load of unit $\mathrm{i}$ and unit j, respectively; $Q_{\text {plan }}(i)$ represents the minimum water consumption that can be optimized by one unit of unit $\mathrm{i}$.

The steps are as follows:

1. The first online unit in the unit sequence is found, and the load of the unit is increased by 1 unit.

2. Another online unit is found. In the unit sequence, the load of this unit is reduced by 1 unit. The water consumption of this scheduling plan is calculated and denoted as $Q_{s}(1,1)$.

3. The previous unit is restored, and the next unit is found in the unit sequence. The load of the selected unit is reduced by 1 unit, and the water consumption of the scheduling scheme is calculated, which is denoted as $Q_{s}(1,2)$.

4. All online units are searched in the same way, and the option with the minimum water consumption is selected, denoted as $Q_{\text {plan }}(1)$. This value represents the water consumption that can be optimized when the first online unit is reduced by one unit.

5. All units are traversed to optimize step by step to get the minimum water consumption optimized for that stage

6. Scheduling table with minimum water consumption has been updated.

7. This method is applied continuously until the optimal load allocation table is obtained.

\subsection{The Flowchart of IBWOA for STHGS}

The IBWOA is applied to solve STHGS problems. The flowchart is shown in Fig. 2.

\section{Case Study}

\subsection{Introduction of Three Gorges Hydropower Station}

The three gorges dam is the world's largest hydropower station and the largest engineering project 
ever built in China. There are 26 mixed-flow generators at the three gorges hydropower station. All of them have a maximum load is $700 \mathrm{MK}$. The five types of hydroelectric units have a total installed capacity of 18,200MW. Table 1 shows the types and operation zones of each unit. Fig.3 shows the characteristic flow curves of five hydro units under $75 \mathrm{~m}, 88 \mathrm{~m}$, and $107 \mathrm{~m}$ water heads.

In this paper, the STHGS problem is set to range for one day at 24 hours. The hourly power demand of the three gorges hydropower station at each period is $7425,7425,7570,8000,8000,8000,8000$, $8000,8000,8000,9317,9754,9026,8881,800,8000,8000,8000,8000,8000,9754,9172,9900$, 9900, 8000, 8000 and $8000 \mathrm{MW}$.

\subsection{Algorithm parameter setting}

The STHGS was calculated by GA, PSO, ACO, WOA, and IWOA, and each algorithm was running 20 times. The $75 \mathrm{~m}, 88 \mathrm{~m}$, and $105 \mathrm{~m}$ respectively represent the low, medium, and high water heads of the three gorges hydropower station.

The parameters of the algorithm are set as follows: QT is 100 , GT is $26, \mathrm{M}$ is 80 , inertia weight $w_{1}=1$, $w_{2}=0.1$, random probability $q_{1}=0.95, q_{2}=1$, perturbation variation probability $P_{m}=0.01$, constant $b=2$. The above parameters are used to solve the mathematical model of economic operation in three gorges hydropower station.

\subsection{Simulation Results and Discussion}

The Proposed IBWOA algorithm was run 20 times in the STHGS model of Three Gorges Hydropower Station. The results show that the optimal total water consumptions corresponding to $75 \mathrm{~m}, 88 \mathrm{~m}$ and $107 \mathrm{~m}$ water heads are $1,058,323,824 \mathrm{~m}^{3}, 892,526,784 \mathrm{~m}^{3}$ and $745,337,067 \mathrm{~m}^{3}$, respectively. The schedule table is shown in Table 2.

According to the data in table 2, the opening or closing time of all units is greater than or equal to $4 \mathrm{~h}$, and the load distribution of these units is in the stable operation area. The results show that the 
minimum uptime/downtime constraint is satisfied by using the minimum uptime/downtime repair strategy. The problem of unsatisfied output can be solved by using spinning reserve capacity constraints. Therefore, these repair methods are effective.

In order to verify the effectiveness of the proposed IBWOA algorithm for the STHGS problems, Genetic algorithm(GA), particle swarm optimization (PSO), ant colony optimization(ACO), whale optimization algorithm(WOA), and IBWOA was run 20 times in the STHGS model of Three Gorges Hydropower Station, and the different algorithms were run 20 times independently under the same initial conditions. Fig.4 shows the convergence characteristics of GA, PSO, ACO, WOA, and IBWOA under different water heads. The results of different algorithms are shown in Table 3.

As shown in Fig. 4, the water consumption calculated by IBWOA is smaller than that obtained by other algorithms under the three water heads. When the water head is $75 \mathrm{~m}$, the convergence process of WOA and ACO is similar, which indicates that WOA can better solve the $0-1$ problem. IBWOA has the fastest convergence rate because the adaptive nonlinear inertia weight is adopted. When the water head is $88 \mathrm{~m}$ or $107 \mathrm{~m}$, the convergence rate of IBWOA, WOA and ACO are similar at the early stage of iteration. All three algorithms search for the optimal solution quickly. However, after 50 iterations, ACO and WOA both fall into the local optimum, while IBWOA jumps out of the local optimum, which because the improving random search method and the mutation mechanism are adopted.

According to Table 3, we can see that the results of WOA are significantly better than GA and PSO, which shows the powerful optimization ability of WOA. Comparing WOA with ACO with a strong ability to solve 0-1 problem, we found that most of the results of BWOA were better than ACO, which indicated that WOA had excellent ability to solve 0-1 problem. However, the standard deviation of WOA is far lower than that of other algorithms. Although this proves the stability of WOA, it also 
reflects its characteristic of easy falling into the local optimum. The results of IBWOA are lower than those of other algorithms under several water heads, which proves its better optimization ability. All these indicate that the improvement of IBWOA is successful, which improves the algorithm's optimization ability and improves the algorithm's ability to jump out of the local optimum.

In Section 5.4, a locally balanced dynamic search mechanism is proposed. The number of iterations of all algorithms was 80 , and then the number of iterations of the locally balanced dynamic search mechanism was 20. Table 4 shows the improvement effect of locally balanced dynamic search mechanism. The results in Table 4 confirm our guess that a stable optimal table does not yield the best load distribution. When the water head is $75 \mathrm{~m}$, the results calculated by WOA according to the stable optimal table are optimal. However, all the results can be further optimized in other cases. So the Proposed A strategy helps solve the STHGS problem.

\section{Conclusions}

This paper has introduced an IBWOA to solve the STHGS problem. The spatial optimal load distribution is combined with the temporal unit commitment combination model, and the binary array is used to represent the start/stop state of the unit. The whale algorithm's search mechanism is optimized, and the inertia weight and perturbation variation strategy are introduced to improve the algorithm's optimization ability. The UC subproblem was solved by two repair strategies, and the ELD subproblem was solved by an OSLDT. The Locally balanced dynamic search mechanism improves the non-optimal condition of the scheduling table caused by the repair mechanism. The comparison between the IBWOA algorithm and other algorithms shows that the average water consumption and optimal water consumption calculated by IBWOA are the lowest. In summary, the IBWOA proposed in this paper effectively solves the STHGS problems, and a novel and effective way is provided for solving STHGS problems. 


\section{Declarations}

Ethics Approval: Not applicable.

Consent to Participate: Not applicable.

Consent to Publish: Not applicable.

Authors Contributions: Conceptualization and material preparation: Kan Yang; Methodology, data collection, modelling process and analysis: Kun Yang.

Funding: The achievement is supported by the National Key Basic Research Program of China (973 Program) (2012CB417006) and the National Science Support Plan Project of China (2009BAC56B03).

Competing Interests: None.

Availability of data and material: Not applicable.

Code Availability: Not applicable.

\section{References}

Cai JJ, Ma XQ, Li LX, Peng HP (2007) Chaotic particle swarm optimization for economic dispatch considering the generator constraints. Energy Convers Manag 48(2):645-653

Catalão JPS, Mariano SJPS, Mendes VMF, Ferreira LAFM (2006) Parameterisation effect on the behaviour of a head-dependent hydro chain using a nonlinear model. Electr Power Syst Res 76(6-7):404-412

Chen HL, Guo RP (2011) Unit commitment based on improved discrete particle swarm optimization. Power System Technology 35(12):94-99

Cheng C-P, Liu C-W, Liu C-C. Unit commitment by Lagrangian relaxation and genetic algorithms. IEEE Trans Power Syst 2000;15:707-14.

Cristian Finardi E, Reolon Scuzziato M. Hydro unit commitment and loading problem for day-ahead operation planning problem. Int J Electr Power Energy Syst 2013;44:7-16.

Dudek G. Adaptive simulated annealing schedule to the unit commitment problem. Electr Power Syst 
Res 2010;80:465-72.

Fakhar MS, Kashif SAR, Saqib MA, Hassan TU (2015) Non cascaded short-term hydro-thermal scheduling using fully-informed particle swarm optimization. Int J Electr Power Energy Syst 73:983-990

Feng ZK, Niu WJ, Cheng CT, Wu XY (2018) Peak operation of hydropower system with parallel technique and progressive optimality algorithm. Int J Electr Power Energy Syst 94:267-275

Finardi EC, Scuzziato MR (2013) Hydro unit commitment and loading problem for day-ahead operation planning problem. Int J Electr Power Energy Syst 44(1):7-16

Hu F, Zhang DH, Y ang XC (2012) Optimal load distribution and commitment of automatic generation control units in hydropower station based on ant colony algorithm. Water Resources and Power 30(12):123-126

Hu H, Y ang K, Liu L, Su LW, Y ang Z (2019) Short-term hydropower generation scheduling using an improved cloud adaptive quantum-inspired binary social spider optimization algorithm. Water Resour Manag 33(7):2357-2379

Jabr RA, Coonick AH, Cory BJ (2000) Homogeneous linear programming algorithm for the security constrained economic dispatch problem. IEEE Transactions on Power System 15(3):930-936

Jukna S (2014) Limitations of incremental dynamic programming. Algorithmica 69(2):461-492

Kumar R, Sharma D, Sadu A (2011) A hybrid multi-agent based particle swarm optimization algorithm for economic power dispatch. Electrical Power and Energy System 33(1):115-123

Labadie JW (2004) Optimal operation of multireservoir systems: state-of-the-art review. J Water Resour Plan Manag 130(2):93-111

Lu B, Li K, Zhang H, Wang W, Gu H (2013) Study on the optimal hydropower generation of Zhelin reservoir. J Hydro-environ Res 7(4):270-278

Mirjalili S, Lewis A (2016) The whale optimization algorithm. Adv Eng Software 95:51-67.

Nilsson O, Sjelvgren D. Hydro unit start-up costs and their impact on the short term scheduling strategies of Swedish power producers. IEEE Trans Power Syst 1997;12:38-44 
Ohishi T, Santos E, Arce A, Kadowaki M, Cicogna M, Soares S. Comparison of two heuristic approaches to hydro unit commitment. Power Tech, 2005 IEEE Russia. IEEE; 2005. p. 1-7.

Ovidiu Ivanov, Bogdan-Constantin Neagu, Gheorghe Grigoras, et al. Optimal Capacitor Bank Allocation in Electricity Distribution Networks Using Metaheuristic Algorithms. 2019, 12(22)

Peng L, Zhou J, Wang C, Qiao Q, Li M (2015) Short-term hydro generation scheduling of xiluodu and xiangjiaba cascade hydropower stations using improved binary-real coded bee colony optimization algorithm. Energy Conv Manag 91(91):19-31

Pérez-Díaz JI, Wilhelmi JR, Arévalo LA. Optimal short-term operation schedule of a hydropower plant in a competitive electricity market. Energy Convers Manage 2010;51:2955-66.

Saraiva JT, Pereira ML, Mendes VT, Sousa JC. A simulated annealing based approach to solve the generator maintenance scheduling problem. Electr Power Syst Res 2011;81:1283-91.

Senthil Kumar V, Mohan M. Solution to security constrained unit commitment problem using genetic algorithm. Int J Electr Power Energy Syst 2010;32: 117-25.

Shang Y, Fan Q, Shang L, Sun Z, Xiao G (2019b) Modified genetic algorithm with simulated annealing applied to optimal load dispatch of the Three Gorges Hydropower Plant in China. Hydrol Sci J 64(9):1129-1139

Shi, L., Hao, J., Zhou, J., \& Xu, G. (2004). Short-term generation scheduling with reliability constraint using ant colony optimization algorithm. Intelligent Control and Automation, 2004. WCICA 2004. Fifth World Congress on (V ol.6, pp.5102-5106 V ol.6). IEEE

Siu TK, Nash GA, Shawwash ZK. A practical hydro, dynamic unit commitment and loading model. IEEE Trans Power Syst 2001;16:301-6.

Su LW, Yang K, Hu H, Yang Z (2019) Long-term hydropower generation scheduling of large-scale cascade reservoirs using chaotic adaptive multi-objective bat algorithm. Water 11:2373. https://doi.org/10.3390/w11112373

Vaisakh K, Srinivas L. Evolving ant colony optimization based unit commitment. Appl Soft Comput 2011;11:2863-70. 
Wang Dingyi, Wu Yonggang. Automatic generation control for gezhouba erjiang hydropower plants[J].Proceedinng of the 10th IEEE Region Conference on Computer, Communication, Controlm and Power Engineering,1993,(1):59-63

Wood AJ, Wollenberg B (1996) Power generation operation and control - 2nd edition. IEEE Power Energy Mag 12(4):90-93

Wu F (2015) Economic dispatch for wind-thermal hybrid power systems based on particle swarm optimization. North China Electric Power University

Zhao T, Zhao J, Y ang D (2012) Improved dynamic programming for hydropower reservoir operation. J Water Resou Plan Manag 140(3):365-374

Zhe Yang, Kan Yang, Lyuwen Su, et al. The Short-Term Economical Operation Problem for Hydropower Station Using Chaotic Normal Cloud Model Based Discrete Shuffled Frog Leaping Algorithm. 2020, 34(3):905-927.

Zheng J, Yang K, Lu X (2013) Limited adaptive genetic algorithm for inner-plant economical operation of hydropower station. Hydrol Res 44(4):583-599 
Figures

\begin{tabular}{|c|c|c|c|c|c|c|c|c|c|c|c|c|}
\hline period & 1th & ${ }^{2 \text { th }}$ & 3th & 4th & 5th & \multirow{6}{*}{$\stackrel{\text { repair }}{\longrightarrow}$} & period & 1th & $2 \mathrm{th}$ & 3th & it th & $3 \mathrm{th}$ \\
\hline 1 & 1 & 1 & 1 & 0 & 1 & & 1 & 1 & 1 & 1 & 0 & 1 \\
\hline 2 & 1 & 0 & 1 & 1 & 1 & & 2 & 1 & 1 & 1 & 0 & 1 \\
\hline 3 & 1 & 1 & 1 & 1 & 0 & & 3 & 1 & 1 & 1 & 1 & 0 \\
\hline 4 & 0 & 1 & 0 & 1 & 1 & & 4 & 0 & 1 & 0 & 1 & 0 \\
\hline 5 & 0 & 1 & 0 & 1 & 1 & & 5 & 0 & 1 & 0 & 1 & 1 \\
\hline
\end{tabular}

Figure 1

Minimum uptime/downtime constraint mechanism diagram

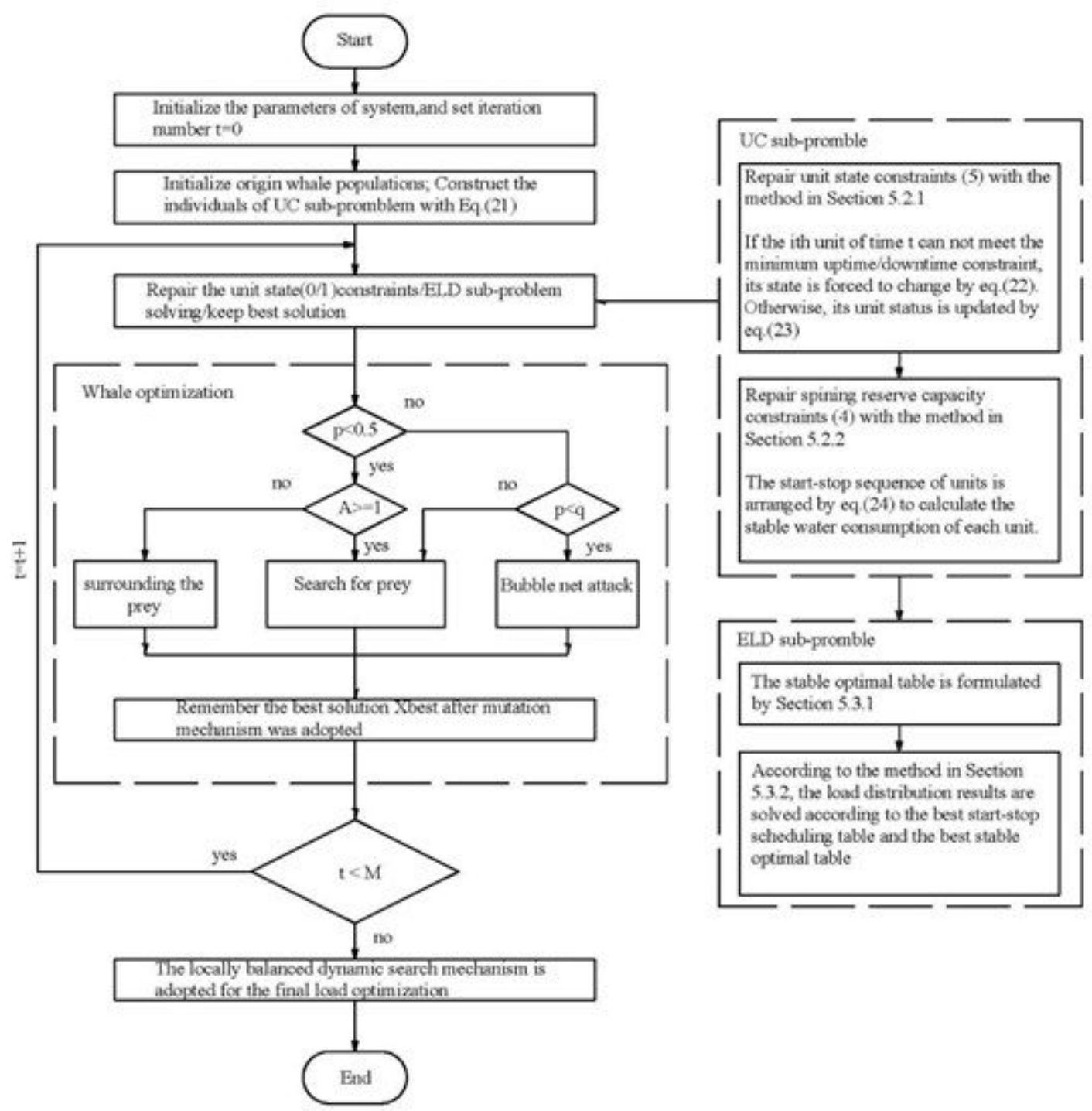


Figure 2

The flowchart of the IWOA for solving the STHGS problem
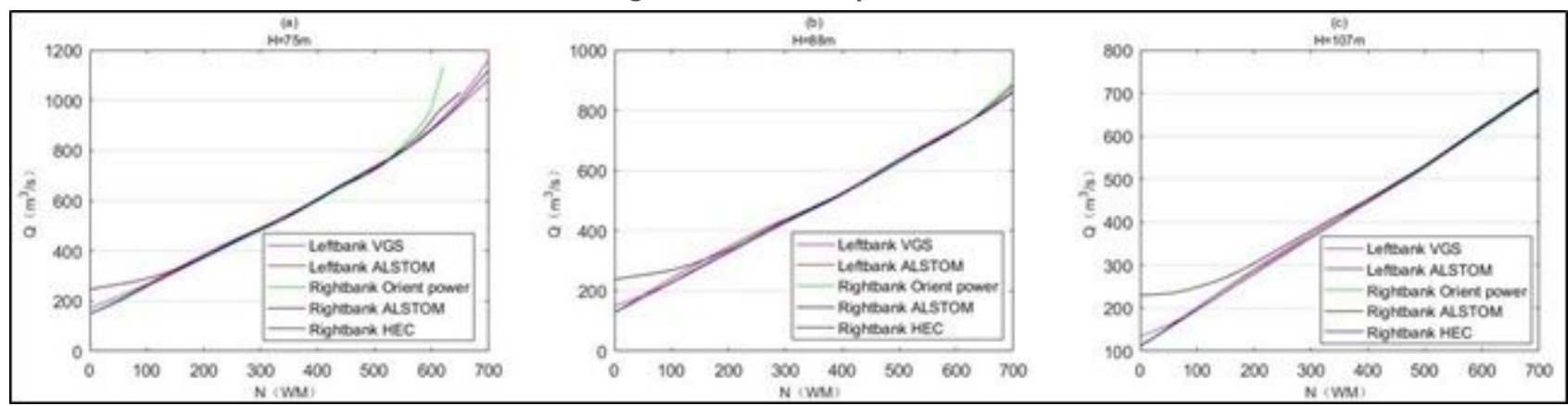

Figure 3

The characteristic curves of different types of units under different water heads 

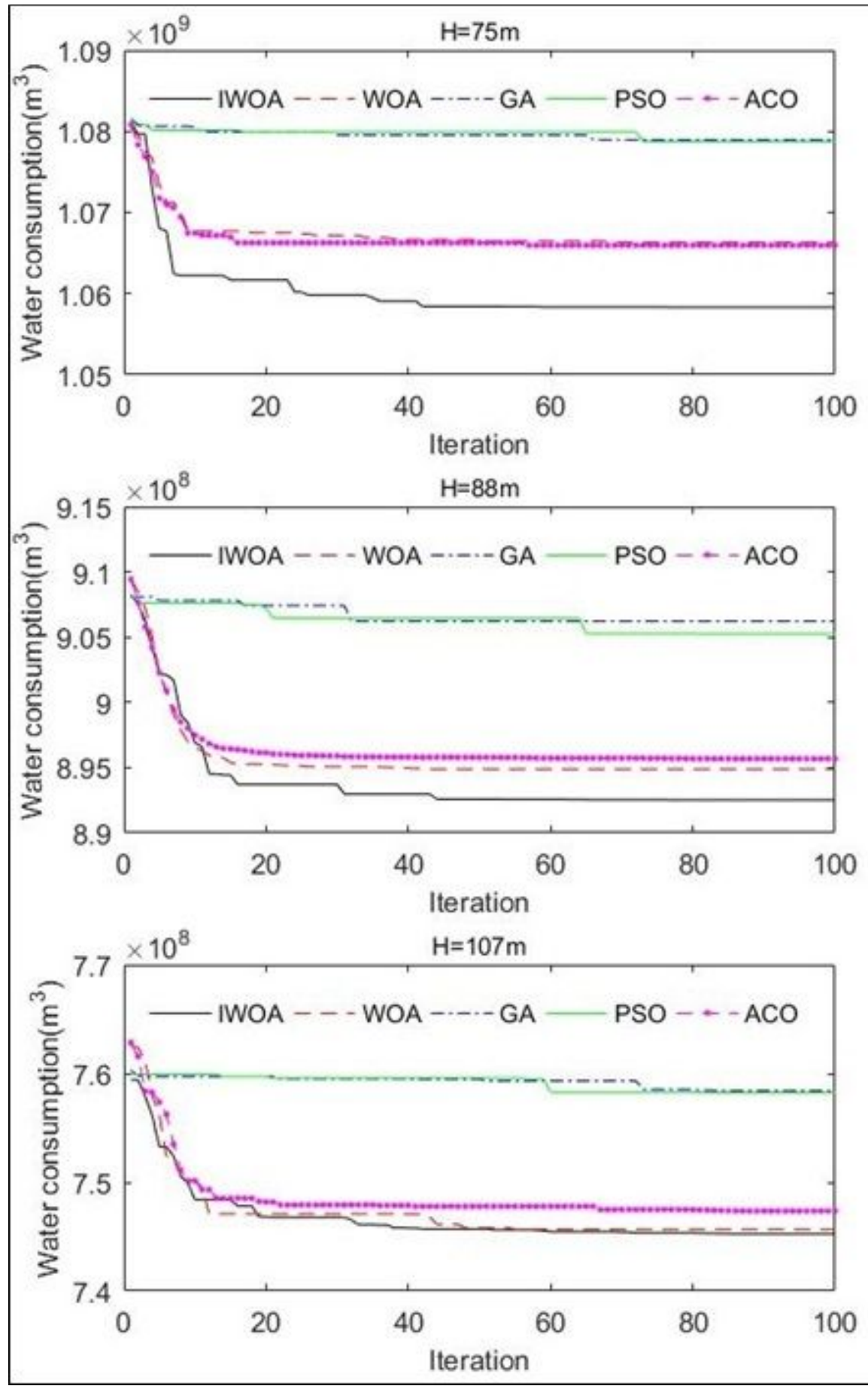

Figure 4

Convergence curves of different algorithms applied to STHGS problem 